\title{
Non-Surgical Reduction of Septal Myocardial Mass in a Patient with Hypertrophic Obstructive Cardiomyopathy
}

\author{
Yuichi Fusegawa, Sumihisa Abe, Mamoru SaKakibara and Shunnosuke Handa
}

\begin{abstract}
Non-surgical reduction of septal myocardial mass was performed with success in a 48-yearold male with hypertrophic obstructive cardiomyopathy. Angioplasty balloon inflated in the first septal branch of the left anterior descending artery dramatically reduced the pressure gradient across the outflow tract of the left ventricle. Following injections of absolute alcohol into the artery with the balloon kept inflated induced a small septal myocardial infarction. By hemodynamic evaluation, the peak pressure gradient of $108 \mathrm{mmHg}$ before the procedure was decreased to $30 \mathrm{mmHg}$. Clinical improvement in New York Heart Association (NYHA) functional class was obtained. At 3-month follow-up, the patient was doing quite well; the gradient was only $10 \mathrm{mmHg}$ at rest and $25 \mathrm{mmHg}$ with Valsalva maneuver.

(Internal Medicine 38: 585-589, 1999)
\end{abstract}

Key words: angioplasty balloon, pressure gradient

\section{Introduction}

In patients with hypertrophic obstructive cardiomyopathy (HOCM), negative inotropic agents such as verapamil and betablockade effectively reduce the pressure gradient across the outflow tract of the left ventricle $(1,2)$. The pharmacological effects, however, are not always sufficient to improve the hemodynamics and symptoms. In the patients with severe obstruction, septal myectomy (3), and mitral valve replacement (4), or implanted dual chamber pacing (5) have been employed as an alternative measures. It was reported recently (6-9) that non-surgical septal reduction of myocardial mass to reduce the pressure gradient with interventional catheterization improves symptoms. Here, we report an experience of a middle aged man with HOCM with intractable episodes of angina and dyspnea on exertion who was successfully treated with the interventional therapy.

\section{Case Report}

The patient was a 48-year-old male with HOCM. He was noted to have a heart murmur in childhood. At age 44 , he started to have episodes of angina with dyspnea on exertion. He was treated by a local doctor, and the symptoms had been progressively worsened to New York Heart Association (NYHA) Class II. He was admitted to Tokai University Hospital for further evaluation. On physical examination, his height was $163 \mathrm{~cm}$, weight $70 \mathrm{~kg}$, and heart rate $70 / \mathrm{min}$. His blood pressure was $130 / 80$. Bisferious pulse was palpable in carotid artery. Peripheral pulses were normal. Pulse of maximal impulse was in the 5th intercostal space on the midclavicular line with left ventricle in systole. S1 was normal. S2 showed physiological split. There was no S3, no S4. There was a systolic ejection murmur of Levine III/VI at the left sternum border. Lungs were clear to auscultation and percussion. Electrocardiogram (ECG) recording revealed normal sinus rhythm with occasional ventricular premature beats, high voltage with giant negative $T$ waves over the left precordial leads indicating marked left ventricular hypertrophy. A chest X-ray revealed cardiomegaly with a prominent 4th left cardiac border. Cardiothoracic ratio (CTR) was $55 \%$. Echocardiogram showed hypertrophied left ventricle with asymmetric septal hypertrophy of more than 1.3. Systolic anterior movement of the mitral leaflets with obstruction of the outflow tract was observed. The left ventricular ejection fraction was $78 \%$. By Doppler echocardiogram, the peak pressure gradient in systole across the outflow tract was $135 \mathrm{mmHg}$.

The patient was started on $50 \mathrm{mg}$ of atenolol qd, $120 \mathrm{mg}$ of verapamil and $100 \mathrm{mg}$ of mexiletine TID, though his symptoms progressively worsened to NYHA Class III. Therefore, informed consent for non-surgical reduction of the septal myocardial mass as an interventional therapy was obtained from the patient as well as from his father and sister.

The cardiac catheterization revealed aortic pressure of $88 /$ $60 \mathrm{mmHg}$, left ventricular pressure of 196/13 $\mathrm{mmHg}$. The peak

From the Division of Cardiology, Department of Internal Medicine, Tokai University School of Medicine, Isehara

Received for publication October 29, 1998; Accepted for publication March 1, 1999

Reprint requests should be addressed to Dr. Yuichi Fusegawa, the Division of Cardiology, Department of Internal Medicine, Tokai University School of Medicine, Bohseidai, Isehara, Kanagawa 259-1193 


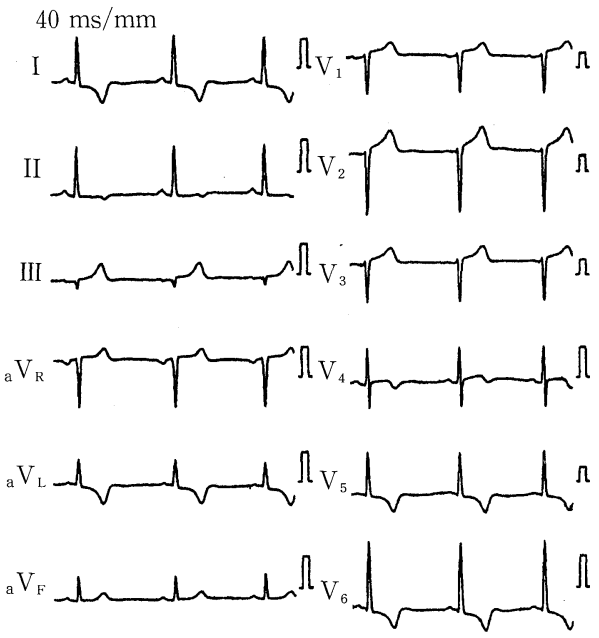

A
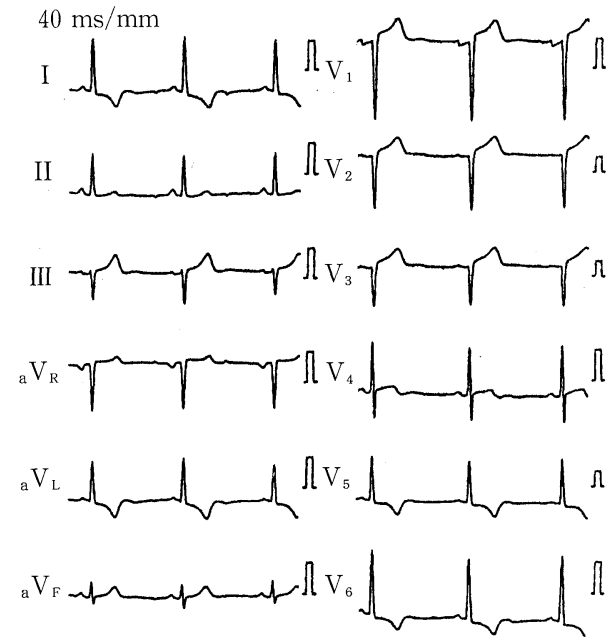

B

Figure 1. Twelve-lead ECG before and after septal reduction treatment. ECG recording showed left ventricular hypertrophy with giant negative $T$ wave (A). After non-surgical septal reduction, small $r$ waves in $\mathrm{V} 2$ and $\mathrm{V} 3$ leads had disappeared (B).

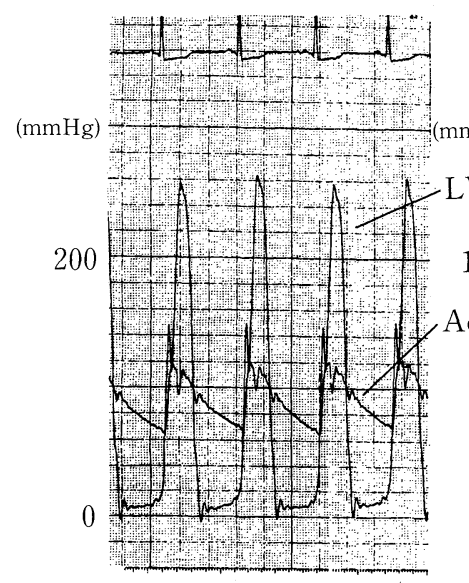

(A) before

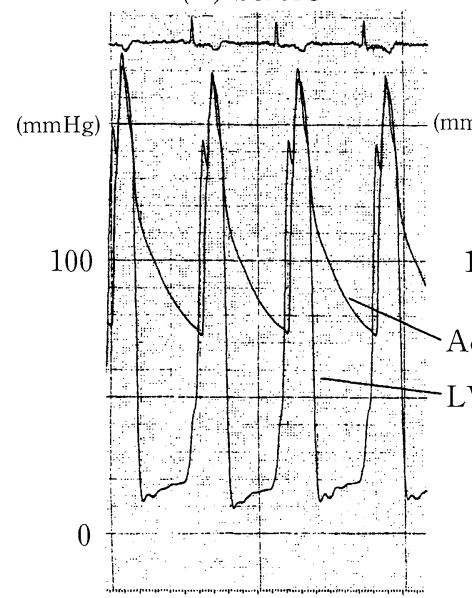

(C) 3-months

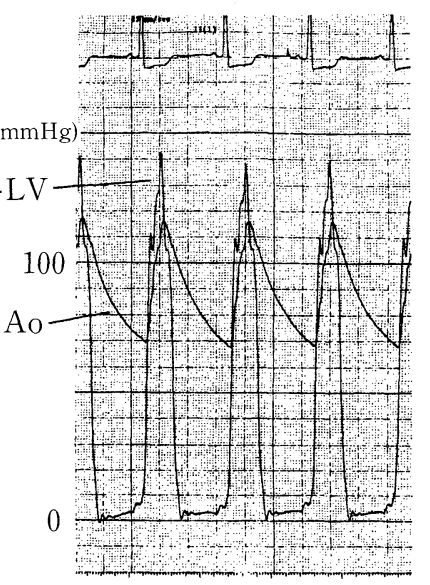

(B) after

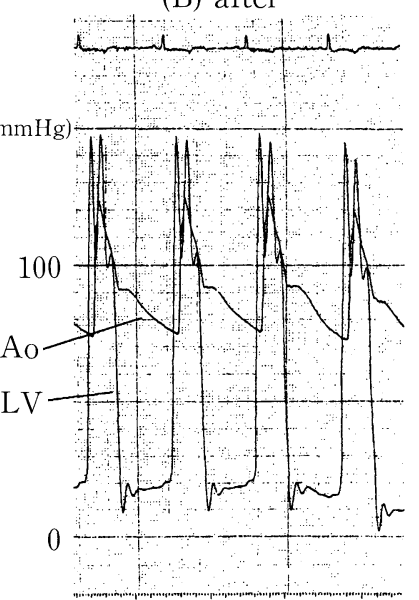

(D) Valsalva maneuver pressure gradient was $108 \mathrm{mmHg}$ in systole. The cardiac output was $3.46 \mathrm{l} / \mathrm{min} / \mathrm{m}^{2}$. A $2.25 \mathrm{~mm}$ angioplasty balloon catheter was placed in the first septal branch of the left anterior descending artery. The occlusion of the artery by temporary balloon inflation resulted in the reduction of the pressure gradient across the outflow tract from 108 to $5 \mathrm{mmHg}$. During the ballooning procedure absolute alcohol of $0.8 \mathrm{ml}$ was injected through the central lumen of the catheter and kept in site for 5 minutes with the balloon inflated. Coronary arteriography confirmed the blockage of the target artery. The procedure was repeated twice. After the 2nd injection, the patient complained to have chest pain. Morphine sulfate of $10 \mathrm{mg}$ intravenous injection was effective to relieve the pain. The final pressure gradient was $30 \mathrm{mmHg}$. Doppler echocardiogram just after the procedure showed the gradient of $32 \mathrm{mmHg}$. Just after the procedure, Magnetic resonance imaging (MRI) showed alcohol stained hypertrophied septum.

The peak rise of creatinine kinase 10th hours after the procedure was $882 \mathrm{IU}$. By ECG monitoring transient junctional rhythm with heart rate of $72 / \mathrm{min}$ and the right bundle branch block were observed. They disappeared on the 10 post-procedure day. The patient tolerated the procedure well and has not since complained of any episode of angina nor dyspnea.

At 3-month follow-up, hemodynamic evaluation revealed the pressure gradient of $10 \mathrm{mmHg}$ across the outflow tract at

Figure 2. Simultaneous pressure recordings from the left ventricular inflow tract and the aorta before (A) and after (B) septal reduction treatment. The left ventricular outflow tract pressure gradient is reduced by the procedure. At the 3-month follow-up, left ventricular outflow tract gradient was reduced to $5 \mathrm{mmHg}$ (C), but the Valsalva maneuver increased it (D). 


\section{Non-Surgical Septal Reduction to HOCM}

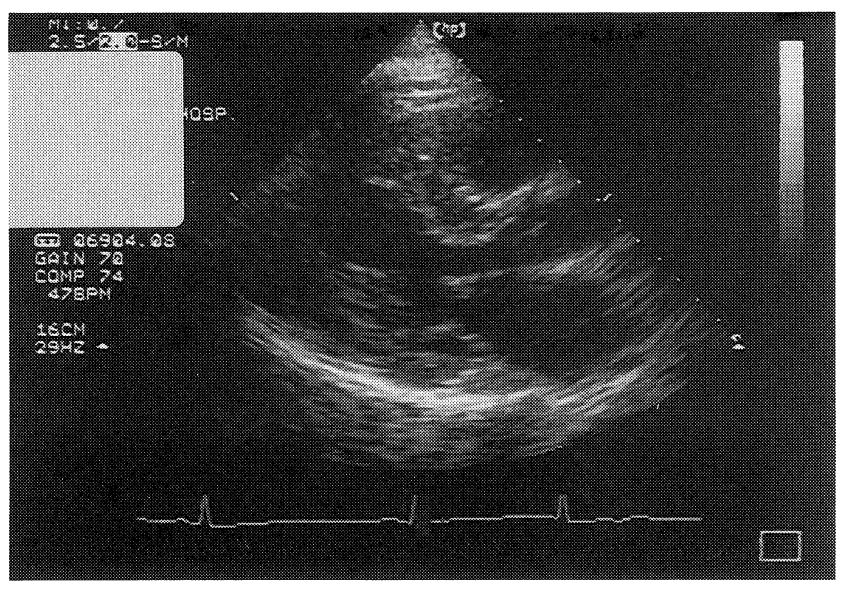

A

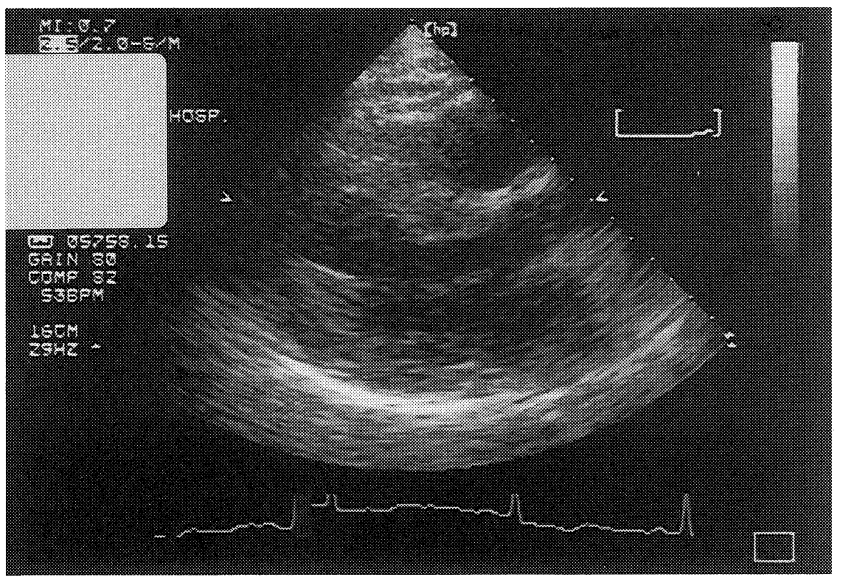

$\mathrm{B}$ rest, the though Valsalva maneuver increased the gradient up to $25 \mathrm{mmHg}$. The left ventricular end-diastolic pressure was $13 \mathrm{mmHg}$. Cardiac output was $3.30 \mathrm{l} / \mathrm{min} / \mathrm{m}^{2}$. ECG recording showed the QS pattern in leads V2 and V3, but a major ST-T change was not shown. On echocardiogram, the left ventricular ejection fraction was $72 \%$ and the systolic anterior movement had disappeared, and on MRI, the thickness of hypertrophied septum was reduced. He was treated by $25 \mathrm{mg}$ of atenolol and $120 \mathrm{mg}$ of verapamil, and his symptoms were improved to NYHA Class II.

\section{Discussion}

A significant number of patients with HOCM may suffer from angina, dyspnea on exertion and syncope (10). There are some distinct strategies for the management of HOCM. The pharmacological measure using a beta-adrenoceptor blockade and the calcium channel blocker verapamil is common, though the effects are not always sufficient to control the symptoms $(2,9)$.

It is known that surgery with septal myectomy and mitral valve replacement are effective to reduce the pressure gradient across the outflow tract of the left ventricle and to manage the clinical symptomatology $(3,4)$. Myotomy with open chest sur-

Figure 3. Echocardiographic images of long-axis view before and after septal reduction. Both pictures were taken at the peak of $R$ wave in ECG monitor. Just before the procedure (A), hypertrophied basal septum was shown. At the 3-month followup (B), the thickness of the basal septum was slightly reduced.

Table 1. Measurement values of hemodynamics and echocardiogram

\begin{tabular}{|c|c|c|c|}
\hline & Before & Immediately after & 3-month follow-up \\
\hline \multicolumn{4}{|l|}{ Hemodynamic evaluation } \\
\hline $\begin{array}{l}\text { heart rate }(\text { beat } / \mathrm{min}) \\
\text { pressure }(\mathrm{mmHg})\end{array}$ & 51 & 50 & 51 \\
\hline aorta & $88 / 50 / 67$ & $107 / 60 / 80$ & $146 / 73 / 96$ \\
\hline left ventricle & $196 / 13$ & $137 / 11$ & $156 / 13$ \\
\hline pulmonary capillary & $24 / 21 / 18$ & & $19 / 17 / 15$ \\
\hline pulmonary artery & $40 / 20 / 27$ & & $38 / 17 / 15$ \\
\hline right ventricle & $40 / 9$ & & $35 / 9$ \\
\hline right atrium & $10 / 6 / 5$ & & $7 / 4 / 3$ \\
\hline cardiac output $\left(l / \mathrm{min} / \mathrm{m}^{2}\right)$ & 3.46 & & 3.30 \\
\hline \multicolumn{4}{|l|}{ Echocardiographic measure } \\
\hline \multicolumn{4}{|l|}{ Doppler echocardiogram } \\
\hline LV-Ao peak systolic PG $(\mathrm{mmHg})$ & 135 & 32 & 25 \\
\hline \multicolumn{4}{|l|}{$\begin{array}{l}\text { M-mode echocardiogram } \\
\text { wall thickness (mm) }\end{array}$} \\
\hline interventricular & 17 & 16 & 18 \\
\hline posterior & 13 & 13 & 14 \\
\hline interventricular/posterior & 1.3 & 1.2 & 1.3 \\
\hline ejection fraction $(\%)$ & 78 & 69 & 72 \\
\hline
\end{tabular}

LV: left ventricular, AO: aorta, PG: pressure gradient. 


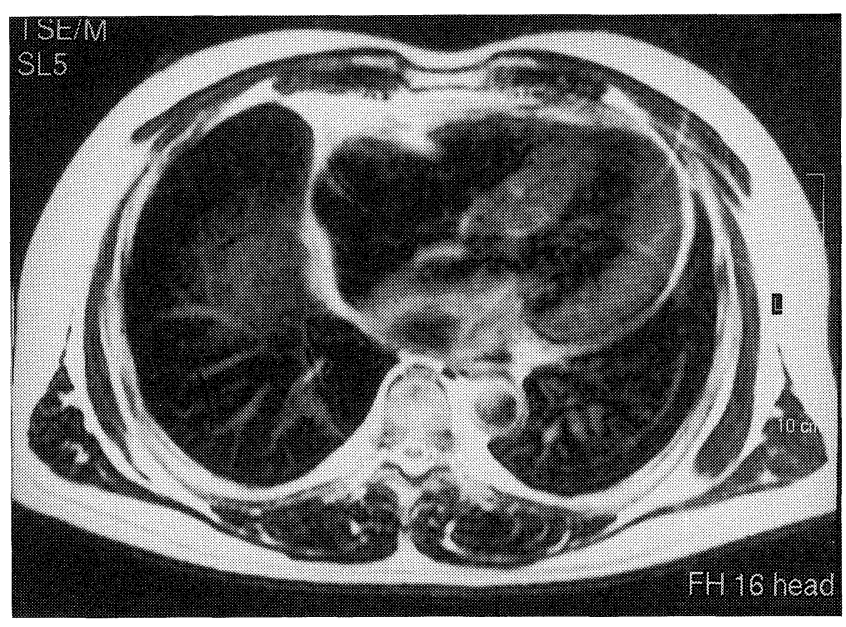

A

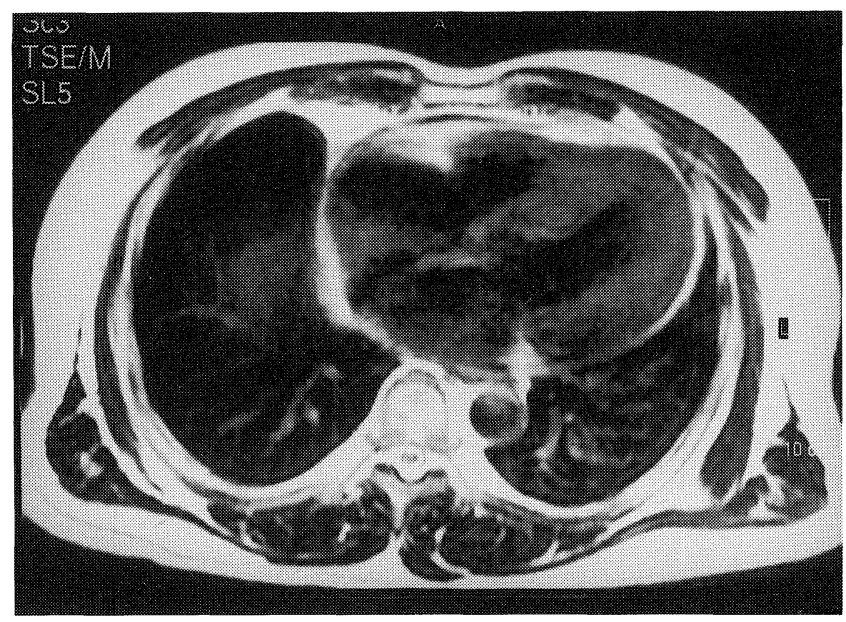

B

Figure 4. Magnetic resonance imaging (MRI) just after septal reduction and at the 3-month follow-up. (A) Just after the procedure, MRI showed alcohol stained hypertrophied septum. (B) At the 3-month follow-up, the thickness of hypertrophied septum is reduced.

gery may contribute to the hemodynamic and clinical benefits, but myotomy remains a complicated operation with surgical risk of death at close to $5 \%$ (9). Dual chamber pacing interferes with narrowing of the outflow tract by shortening the A$\mathrm{V}$ interval and modifying the sequence of ventricular depolarization (5). The outflow gradient can be reduced by the pacing, though, it is not clear whether or not symptomatic benefit can be achieved and the natural history is improved.

Non-surgical reduction of septal myocardial mass with occlusion of the first septal branch of the left anterior descending artery is undoubtedly less invasive and a safer measure with sufficient therapeutic effect to the pathophysiology, compared with surgical myectomy and mitral valve replacement. Eighteen patients were treated with the procedure with success (6). The reduction of pressure gradient across the outflow tract could be confirmed easily with the angioplasty balloon inflation in the septal artery.

Septal infarction may cause acute complications, such as lethal arrhythmia, A-V conduction disturbance and it may chronically develop left ventricular dysfunction with remodeling of the myocardium (11).

In the present case, just after the procedure and at the 3month follow-up, the pressure gradient was almost gone associated with symptomatology. Valsalva maneuver, though, increased the pressure gradient, indicating that a slight obstruction remains.

There was no major complication with this procedure in our patient. The size of septal myocardial infarction was small and the left ventricular function was preserved. There have been several reports (6-8) on the complications of the procedure, such as ventricular arrhythmia including ventricular fibrillation. Seggewiss et al (7) reported complete heart block in $20 \%$ of the patients requiring permanent pace-maker implantation and an 86-year-old woman died with ventricular fibrillation after the successful intervention. Transient occlusion of the left anterior descending artery due to alcohol leaking down the main lumen of the artery was reported (6). The angioplasty balloon must be kept inflated in the septal artery at least for a few minutes after the infusion of absolute alcohol. And, informed consent from the patient is crucial for the procedure.

Although, the non-surgical septal reduction procedure reduces obstruction and improves symptoms, no objective clinical benefit or favorable effect on natural history of HOCM has been documented. More clinical trials including a prospective study are necessary.

\section{References}

1) Harrison DC, Braunwald E, Glick G, Mason DT, Chidsey CA, Ross J Jr. Effects of -adrenergic blockade on the circulation, with particular reference to observations in patients with hypertrophic subaortic stenosis. Circulation 29: 84-98, 1964.

2) Cohen LS, Braunwald E. Amelioration of angine pectoris in idiopathic hypertrophic subaortic stenosis with bete-adrenergic blockade. Circulation 35: 847-851, 1967.

3) Morrow AG, Lambrew CT, Braunwald E. Idiopathic hypertrophic subaortic stenosis, II; operative treatment and the results of pre and postoperative hemodynamic evaluations. Circulation 30: Suppl 4: 120, 4: 151, 1964.

4) Krajcer Z, Leachman RD, Cooley DA, Coronado R. Septal myotomymyomectomy versus mitral valve replacement in hypertrophic cardiomyopathy: ten-year follow-up in 185 patients. Circulation 80: 157-164, 1989.

5) Slade AK, Sadoul N, Shapiro L, et al. DDD pacing in hypertrophic cardiomyopathy; a multicentre clinical experience. Heart 75: 44-49, 1996.

6) Knight C, Kurbaan AS, Seggewiss H, et al. Nonsurgical septal reduction for hypertrophic obstructive cardiomyopathy: outcome in the first series of patients. Circulation 95: 2075-2081, 1997 (see comments).

7) Seggewiss H, Gleichmann U, Faber L, Fassbender D, Schmidt HK, Strick S. Percutaneous transluminal septal myocardial ablation in hypertrophic obstructive cardiomyopathy; acute results and 3-month follow-up in 25 patients. J Am Coll Cardiol 31: 252-258, 1998 (see comments).

8) Tsuchikane E, Nakamura T, Yamazaki K, et al. Transluminal percutaneous septal myocardial ablation in a patient with hypertrophic obstructive cardiomyopathy. Jpn Circ J 62: 537-540, 1998. 


\section{Non-Surgical Septal Reduction to HOCM}

9) Brounwald E. Induced septal infarction, A new therapeutic strategy for hypertrophic obstructive cardiomyopathy. Circulation 95: 1981-1982, 1997 (editorial:comment)(see comments).

10) Braunwald E, Lambrew CT, Rockoff SD, Ross J JR, Morrow AG. Idiopathic hypertrophic subaortic stenosis, I: a description of the disease based upon an analysis of 64 patients. Circulation 30: Suppl 4: 3-119, 1964.

11) Braunwald E, Pfeffer MA. Ventricular enlargement and remodeling following acute myocardial infarction: mechanisms and management. Am J Cardiol 68: 1D-6D, 1991. 